\title{
Study On Application Of Monitoring The Melt Flow Balance Of Injection Molding Process By Using Cavity Pressure Monitoring Technology
}

\author{
Chen Jin-wei* \\ Polymer Processing Engineering Technology \\ Development Center \\ Guangdong Industry Technical College \\ Guangzhou, China \\ e-mail: 205103052@gditc.edu.cn
}

\author{
Yang Ling \\ Dept. of Chemical Engineering \\ Guangdong Industry Technical College \\ Guangzhou, China \\ e-mail: susan.yang@163.com
}

\begin{abstract}
At first this paper described the cavity pressure monitoring technology definition and work principle. Then showed that made use of monitoring the cavity pressure curve and study melt flow balance by specific example. The use of cavity pressure curve could be quickly and accurately get the best process parameters, so change the way of obtaining process parameters optimization from the "experience" basis to "scientific" basis. The article concluded it could understand deeply and grasp the relationship among the three proposals of the cavity pressure curve, process parameters and product quality.
\end{abstract}

Keywords-cavity pressure; injection molding;procedure parameters;melt flow balance

\section{INTRODUCTION}

\section{A. Cavity pressure monitoring technology}

Cavity pressure monitoring technology was an advanced monitoring technology which could monitor the cavity pressure during plastics melt injection molding process in real time and access to cavity pressure curve in recent years. It was a new non-technical experience-dependent and science-based molding process analysis tools following after CAE mold flow analysis technology[1 3].Cavity pressure is not a product size parameters or set process parameters of injection machine that was an actual injection molding procedure parameters [4]. It could be said that cavity pressure monitoring was the monitoring of injection molding procedure parameters, instead of the traditional products quality monitoring by speculating the defect cause by checking products defects which was a passive way. The cavity pressure as Procedure parameters could truly record melt filling cavity process, including injection, holding pressure and cooling stage and the actual melt flow state in the each of cavity which could let us observe visually melt actual flow behavior. According to cavity pressure curve of injection molding process we could analysis the melt actual flow process in the cavity, such as melt flow in the cavity was balanced or not, and what time was imbalance,etc., so that guided us to make a scientific analysis of injection molding process, solve scientifically practical problems.

The traditional methods adjusting the process parameters were based on engineering experience in setting process parameters. Thus, the different engineers were often very different in setting process parameters for the same product.
So that the quality of the products were different. The fundamental reason was that there was no scientific basis to guide people to adjust the process parameters in the process of setting process parameters. Cavity pressure recorded in the melt flow process in the cavity, so according to the cavity pressure curve we could give the scientific guidance in setting process parameters, such as VP switching point was reasonable or not, the reasonableness of holding pressure, holding pressure time was reasonable or not which could be reflected visually by the cavity pressure curve. So we could guide people to adjust scientifically the process parameters by the cavity pressure curve .In the actual production process, even if using the same injection machine and the same set of process parameters to product, but still product quite different quality products, so that it was very difficult that we set correctly process parameters. In fact we knew that as long as the mold and raw material to be determined, then the best cavity pressure curve corresponding to the best products was bound to be determined. When the injection machine was replaced we didn't re-set process parameters based on experience, and it was just ok that according to the cavity pressure curve we set the optimal process parameters. Regardless of how to set the process parameters, as long as the actual cavity pressure curves with the standard cavity pressure curve was the same, corresponding to the same product quality. So we could reduce greatly adjusting machine parameters time.

While cavity pressure could directly reflect the relationship of various molding process parameters. Any change of a molding process parameter was directly reflected by the change of cavity pressure, such as melt temperature, mold temperature, injection pressure, packing pressure, packing time, cooling time etc.change, the cavity pressure would also occur change. These process parameters together determined the quality of products, so that we could think directly cavity pressure determined product quality. In the actual production process, we achieved monitoring of the quality of products by monitoring cavity pressure. When the actual cavity pressure value did not meet the set range, the product must be defective. At this point cavity pressure data acquisition equipment could send digital signals to robot, and separated automatically the defective product, in order to achieve zero-defect production. Also in the production process we could save cavity pressure curve. When the products occurred problems in the production process, we could access the cavity pressure curve saved in the original 
production process, and understand the production conditions and product quality at that time.

\section{B. Cavity pressure monitoring system work principle}

Real-time monitoring cavity pressure could be achieved by the cavity pressure measurement system which included the cavity pressure sensors, data acquisition and processing devices and software system, shown in Figure 1.

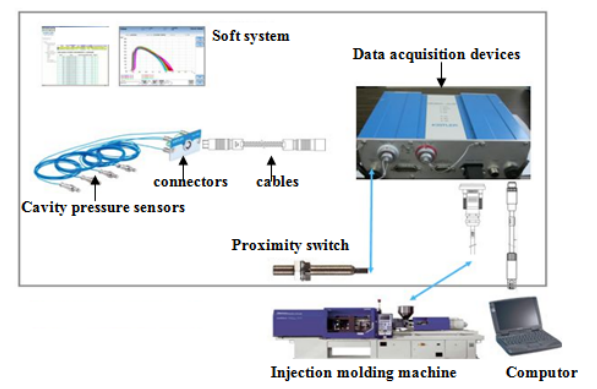

Figure 1. Schematic diagram of cavity pressure monitoring system

Cavity pressure sensor was the main unit of all the monitoring system which monitored the cavity pressure data by using piezoelectric principle [5]. The quartz could generate charge because of the role of mechanical load. The charge amount was proportional to mechanical loads value, which was well-known piezoelectric principle. As the quartz crystal with a high temperature, high natural frequency, high stiffness and other characteristics, and therefore quartz piezoelectric sensor as sensitive components was suitable for measuring cavity pressure.

\section{PREPARATION BEFORE THE EXPERIMENT}

\section{A. Sensor installated location}

When determining the specific location of installing the sensor, we usually based on the characteristics of the product and the mold. If the purpose of mounting the sensor was to try-mold analysis and make process parameters optimization, recommended the sensor could be installed in the near gate location. As be seen from Figure 2, the more close to the gate, the larger the cavity pressure was, the longer the measurement time was, more clearly reflected the melt filling process. If the purpose of mounting the sensor was to solve the shortcomings products part appeared, such as short shot, recommended the sensor installed in the location of products defects. If the products did not have room for sensor installed in defective position, it was recommended that the sensor should be installed near the location of defects. Mold flow analysis could also identify the location of product defects, so the sensor was installed in the location by mold flow analysis.

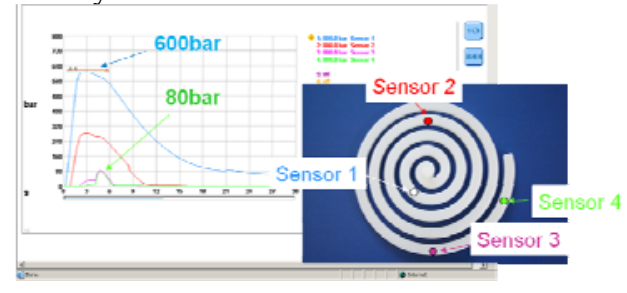

Figure 2. Sensor installated location on the impact of the measured values

As mentioned earlier in this experiment the sensors were installed in the near gate location, shown in Figure 3.

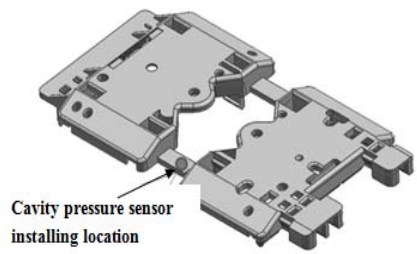

Figure 3. Cavity pressure sensor location diagram

\section{B. The experimental plastic parts}

This experiment illustrated that the cavity pressure monitoring techniques was in the application of monitoring the melt flow balance by a specific case. Figure 4 was an automobile oil pump parts. Raw materials were POM (POM C13031), the United States Hearst Corporation produced. The mold had four cavities with hot runner. The product characteristics were complex structure, heterogeneous thickness of products and many openings and snaps.

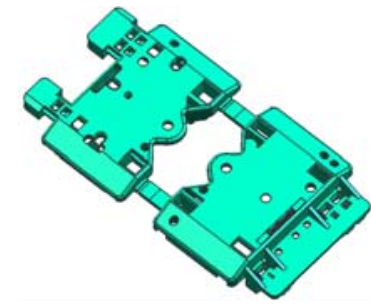

Figure 4. Automobile oil pump parts

During the test mold we could find the melt filling the four cavities was imbalance shown in Figure 5. it could not detect products defects reasons adjusting the process parameters for many times by the experience, and then solved product quality problems. Taking into the strict requirements on the quality of auto products, so decided to use the cavity pressure monitoring technology to do a scientific analysis of the molding process in order to identify product defects cause and solve the problem.

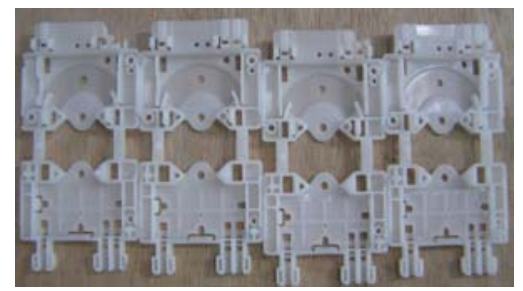

Figure 5. Large differences in product weight of four cavity 


\section{The experimental device}

In this experiment four cavity pressure sensors (diameter $1 \mathrm{~mm}$ ) were installed in the same location of each cavity. Data acquisition system was built-in 8-channel charge amplifier and A / D conversion module which could capture eight pressure signals and four cavity temperature signals. data acquisition software MIS had powerful analysis capabilities that could automatically save the cavity pressure curve which could be superimposed with different periods on the same screen. While the curve data could be exported to EXCEL format, etc.The cavity pressure data acquisition system was shown in Figure 6.

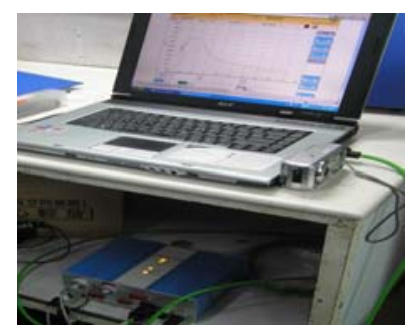

Figure 6. Cavity pressure data acquisition system

\section{EXPERIMENTAL RESULTS AND DISCUSSION}

After installing the sensor, connected the data acquisition system to collect cavity pressure data. Figure 7 showed the four cavity pressure curve less overlap and different cavity pressure in the cavity was quite different. According to these curves, we could determine intuitively and scientifically the melt flow imbalance, so we could know the process parameters and mold structure needed to be improved or not.

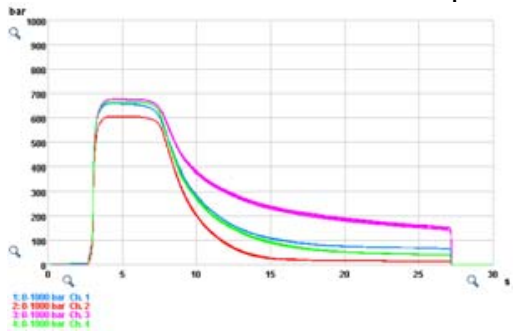

Figure 7. the acquisition of cavity pressure curve before optimizing process parameters (four curves corresponded to four cavity pressure signal, the same below)

Melt flow imbalance had two main reasons. Firstly process parameters were not optimized. Secondly mold structural had some problems. So at first it could improve the flow balance by adjusting the process parameters. But in any case adjusting the process parameters, the melt flow is always unbalanced; we could use the following solutions:

1) Optimizing the mold structure;

2) Improving the injection machine control system;

3) Optimizing the hot runner system and controlling accurately each cavity;
4) Controlling accurately mold temperature;

5) With the help of the cavity pressure curve optimizing deeply the process parameters and hot runner parameters. Figure 8 showed the four cavity pressure curve after optimizing by using above solutions.

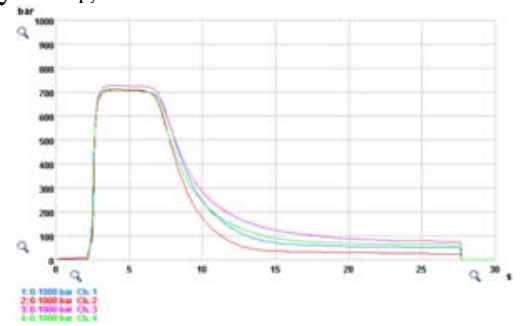

Figure 8.The four cavity pressure curve after optimizing

Compare Figure 7 with Figure 8, the four cavity pressure curve overlap was poor before optimizing, indicating that the melt flow was imbalance, and then the four cavities had large differences in product weight; After optimizing, four cavity pressure curve in the coincidence was better (especially at the maximum value), indicating that the melt flow was balance, the four products in the cavity was almost the same weight.

\section{CONCLUSION}

According the above application cases of the cavity pressure monitoring technology, we could draw the following conclusions and recommendations:

1) By use of cavity pressure curve we could monitor visually the melt flow was balanced or not, which could achieve a good understanding of multi-cavity melt flow balance of the mold cavity.

2) Cavity pressure was injection molding process procedure parameters which was closely related with various molding process parameters. By use of cavity pressure monitoring technology we could get the cavity pressure curve and provide a scientific basis for adjusting process parameters. We could optimize quickly and accurately the process parameters by using cavity pressure curve.

3) After process parameters were optimized the cavity pressure curve was smooth and products had the best quality, while producing process was the most stable. In this time the molding process parameters were optimized parameters. We could save this best cavity pressure curve as a reference cavity pressure curve. In different machines to produce we set the process parameters by using the saved cavity pressure curve as a standard referred curve,we could save a lot of adjusting time.

We knew mold was a black box .So the naked eye could not see the process of the melt filling and any changes in the melt state. The melt filling process directly determines the quality of products, good or bad. By Using cavity pressure monitoring technology, we could visualizes melt filling process, accurately reflect melt state in the injection stage, the compaction stage, holding pressure and cooling stage, so 
mastered the melt flow in the cavity, and helped us to analyze scientifically problems and solve scientifically problems. By using cavity pressure curve monitoring technology we could study the various molding process parameters influence on product performance and solve the problem of adjusting practical production process parameter, so provided us with a more scientific solution to the problem. Hope that in future we could research system the impact on the cavity pressure curve of injection speed, VP switching point, packing pressure, packing time, cooling time, melt temperature, mold temperature and the material ,so deepened the understanding of the relationship among the cavity pressure technology, process parameters and product quality.

\section{ACKNOWLEDGMENT}

This research was supported by Doc. Xu Bai-ping support and help, to express my sincere thanks.

\section{REFERENCES}

[1] Chen Jinwei,"The application of cavity pressure profile in the injection molding process parameters optimization,"Engineering plastics applications , Vol.38, pp.28 31,March 2010.

[2] Zheng Rongcheng,"Mold cavity pressure monitoring techniques,”Abroad plastic,Vol.25,pp.62 66,June 2005.

[3] Zheng Rongcheng,"Mold cavity pressure monitoring and optimization of injection molding processes are zero-defect componts,"Abroad plastic,Vol.27,pp.76 78,May 2007.

[4] Wang Xing Tian,Injection Molding Technology.Chemical Industry Press,2004.

[5] Bernhard Bill. Measuring with Crystals. Germany: verlag moderne industrie, 2002. 